\title{
Early Outcomes of Medial Pivot Total Knee Arthroplasty Compared to Posterior-Stabilized Design: A Randomized Controlled Trial
}

\author{
Vikas Kulshrestha, MS, Munish Sood, DNB, Sarang Kanade, MS*, Santhosh Kumar, DNB ${ }^{\dagger}$, \\ Barun Datta, $\mathrm{MS}^{\ddagger}$, Gaurav Mittal, $\mathrm{MS}^{\S}$ \\ Department of Orthopaedics, Command Hospital, Chandigarh, \\ ${ }^{*}$ Department of Orthopaedics, Dr Shyama Prasad Mukherjee Hospital, Lucknow, \\ ${ }^{\dagger}$ Department of Orthopaedics and Joint Replacement, Command Hospital Air Force, Bengaluru, \\ ${ }^{\ddagger}$ Department of Orthopaedics, Joint Replacement Centre, Army Hospital R \& R, New Delhi, \\ ${ }^{\S}$ Department of Orthopaedics, Military Hospital, Kilkee, India
}

Background: The indications for total knee arthroplasty (TKA) have been expanded to include younger, demanding patients. Some TKA patients expect a return to high-performance activities to restore optimum quality of life. The concept of the medial pivot (MP) TKA is that more natural knee kinematics can be achieved by altering the bearing design. In the present study, we compared the early outcomes of MP TKA with posterior-stabilized (PS) TKA in terms of patient-reported outcomes, function, and performance.

Methods: This randomized study was performed in a high volume joint replacement facility of a tertiary care military hospital. We enrolled 40 patients each in the MP group and PS group and assessed knee flexion, patient-reported outcome (new Knee Society Score [new KSS]), patient performance (Delaware Osteoarthritis Profile Score [DOPS]), and function (Forgotten Joint Score [FJS]) at 2 years after surgery.

Results: Compared to PS group patients, MP group patients had similar patient-reported outcomes assessed by new KSS (satisfaction, expectation, and activity scales) and FJS. MP knee patients had better performance in the timed up and go test ( $p<0.026)$ and self-paced walk test $(p<0.002)$ of DOPS. The gain in knee flexion $\left(9.3^{\circ} \pm 14^{\circ}\right)$ compared to baseline was significantly greater in the PS group $(p<0.013)$.

Conclusions: When assessed by DOPS, getting up from chair and walking speed were significantly better in MP knee patients than in PS knee patients. However, considering the predictable rollback ensured by cam and post, the PS knee produced better knee flexion. Despite these results, patients were equally satisfied with the two designs.

Keywords: Total knee arthroplasty, Medial pivot, Posterior stabilized, Patient performance, Patient-reported outcomes

The overwhelming success of total knee arthroplasty (TKA) has rapidly increased its utilization. ${ }^{1,2)}$ Initially, the procedure was largely meant to relieve pain and preserve

Received September 28, 2019; Accepted November 21, 2019

Correspondence to: Munish Sood, DNB

Department of Orthopaedics, Command Hospital (WC), Chandimandir,

Chandigarh 134107, India

Tel: +91-9876411288

E-mail: soodmunishafmc@gmail.com mobility in the elderly, but it has expanding the spectrum of usage to include a younger, demanding population. Its significant effect on improving quality of life has made enormous socioeconomic impact. To optimize outcomes of TKA, there have been rapid advances in each aspect of the surgery, including preoperative patient optimization, precise surgical technique, effective pain control, and expeditious rehabilitation. ${ }^{2)}$ Even with these changes, $10 \%-15 \%$ of patients remain dissatisfied. ${ }^{3)}$ One of the reasons for dissatisfaction is presumed to be altered kinemat- 
Kulshrestha et al. Medial Pivot vs. Posterior-Stabilized Total Knee Arthroplasty

Clinics in Orthopedic Surgery • Vol. 12, No. 2, $2020 \bullet$ www.ecios.org

ics of the prosthetic knee joint. Seeking improved patient satisfaction and better functional outcomes, the search for an implant that could offer these benefits without compromising longevity continues.

The medial pivot (MP) fixed-bearing prosthesis has a single radius femur and an asymmetric tibial insert, which has a highly congruent medial compartment that offers stability and a less conforming lateral compartment that allows posterior rollback. ${ }^{4)}$ The MP knee system addresses the paradoxical anterior femoral translation encountered by posterior-stabilized (PS) and cruciate-retaining (CR) knees and it provides stability throughout the arc of motion by its inherent design physiognomies. MP TKA has been shown to be associated with greater patient satisfaction. ${ }^{5)}$ However, to date, there is limited evidence to support the use of MP design over other knee designs..$^{6-13)}$ Our study is one such attempt to compare a MP design with a PS design. In the present study, we intend to compare patient-reported outcomes, function, and performance in patients undergoing TKA using a MP knee (ADVANCE, Wright Medical Technology, Arlington, TN, USA) (Fig. 1A) or a PS knee (NexGen Legacy, Zimmer, Warsaw, IN, USA) (Fig. 1B) by using new Knee Society Score (new KSS), Forgotten Joint Score (FJS), and Delaware Osteoarthritis Profile Score (DOPS). ${ }^{14)}$

\section{METHODS}

\section{Study Design}

The study is a randomized, comparative trial with parallel assignment.

\section{Sample Size}

Literature review brings out that the minimum clinically relevant difference in function subscale of new KSS is $8-10$ points. ${ }^{15)}$ The same was used as our primary outcome to calculate the sample size by using Stata ver. 12 (StataCorp., College Station, TX, USA). With one-sided alpha error of 0.05 , to have $80 \%$ power to find any clinically relevant difference in outcome, the required sample size would be 36 patients in each group. To allow for $10 \%$ loss to follow-up, we planned to enroll 40 patients in each group.

\section{Enrollment \& Randomization}

We obtained ethical committee approval (IRB No._CH/ Lko/Jun/16) before patient enrollment. From January 2016 to December 2016, all patients visiting our tertiary care hospital with advanced bilateral osteoarthritis of the knee and planned for TKA were subjected to extensive preoperative workup as published in an earlier study on simultaneous bilateral TKA ${ }^{16)}$ and screened for participation in the study. After optimization of health, they were referred for preanesthetic checkup. All those deemed fit to undergo bilateral TKA were offered to participate in the study. The study nurse (PS) explained the study to the patient, describing the features and advantages of each implant design. Patients were given a brief summary of the study and the need for follow-up if they were enrolled. All consenting patients with primary osteoarthritis were included. Excluded were patients with rheumatoid arthritis or severe deforming arthritis requiring specialized implants and patients who were not available for follow-up for at least 2 years. Demographic details and medical and clinical history of the patients were noted. A trained phys-

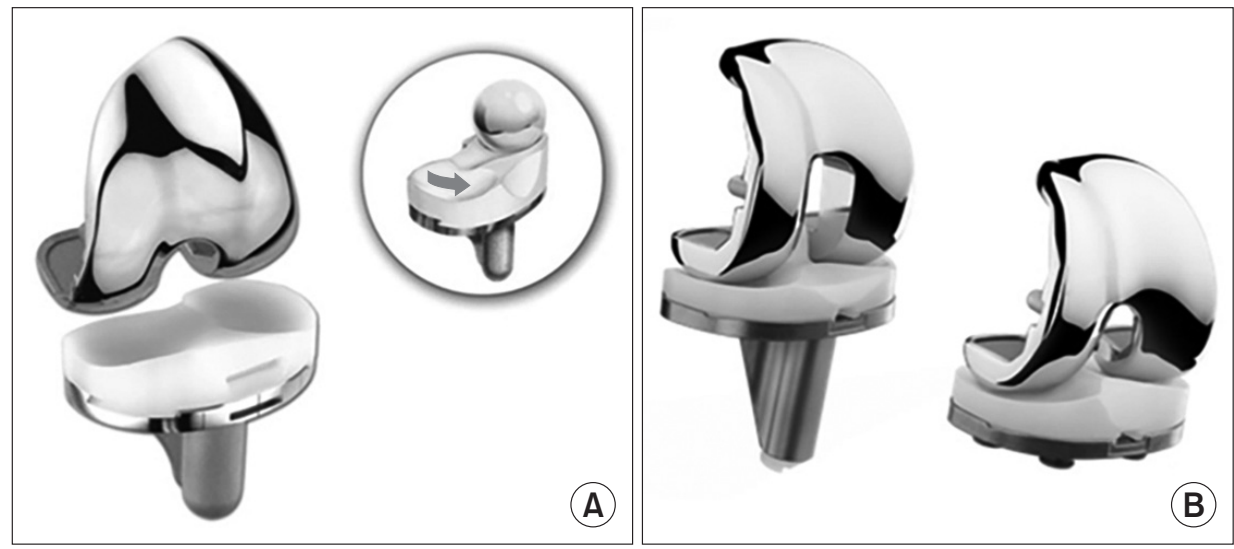

Fig. 1. (A) Photograph of the medial pivot knee showing a deeper and more medial surface of the articular insert that articulates with a congruent medial femoral condyle and the anterior lip that provides stability in flexion and a less congruent lateral surface that allows free movement of lateral femoral condyle along an arcuate path. (B) Photograph showing the posterior-stabilized design with a cam and post mechanism with symmetrical, minimally conforming, articular insert and a post to engage the cam in the femoral condyle and ensure rollback. 
Kulshrestha et al. Medial Pivot vs. Posterior-Stabilized Total Knee Arthroplasty

Clinics in Orthopedic Surgery • Vol. 12, No. 2, $2020 \bullet$ www.ecios.org

iotherapist (AS) using new KSS, FJS, and DOPS assessed the functional status of patients. Patients were randomized into PS or MP group by using computer generated block randomized sequence, implemented by the study nurse using sealed envelopes.

\section{Arthroplasty Protocol}

All patients underwent preoperative workup and optimization of comorbidities. A dedicated counselor (AS) offered preoperative education to all patients regarding preparation for surgery and aftercare including rehabilitation. Patients used overnight chlorhexidine wipes 3 days before surgery. On the morning of surgery, multimodal oral pain control drugs were administered and continuously given postoperatively. All the operations were performed under single-shot, low-dose, spinal anesthesia. At induction, we used broad-spectrum antibiotics and injected tranexamic acid. The administration of prophylactic antibiotics was continued for 24 hours. Additional dose of tranexamic acid was given at 2 hours after surgery, and injectable steroid (injection of dexamethasone) was given once daily for 48 hours. A fellowship-trained surgeon (VK) performed all operations under tourniquet control on the patient under the same anesthesia. Medial parapatellar arthrotomy was used in all operations. TKA was carried out by using ADVANCE MP Knee System or NexGen Legacy PS system as per randomization. We used gap-balancing principles in both groups. Femur sizing was done by using a posterior referencing jig in MP TKA and an anterior referencing jig in PS TKA. Femur rotation was set to $3^{\circ}$ in most cases. All attempts were made to size the femoral component to avoid any overhang. We used the femoral component size, which restored the posterior femoral offset without anteriorly notching or overstuffing the patellofemoral joint. In the PS knee, the posterior cruciate ligament (PCL) was sacrificed along with the notch bone cut in all cases. In MP TKA, we resected the PCL only if we could not achieve flexion balance or if the flexion gap was too tight and extension gap was acceptable. The tibia was cut at a $3^{\circ}$ slope in MP TKA as compared to $7^{\circ}$ in PS TKA as per the described technique for each instrumentation. In none of the cases, we resurfaced the patella. However, we performed patelloplasty in all cases by shaving the margins of patella to make it more conform to the implant. We also resected the lateral edge of the patella and cauterized the lateral border of the patella. In all cases, we used barbed bidirectional absorbable sutures for closure of arthrotomy. No drains were used. Postoperatively, all patients received adductor canal block for pain relief. Deep vein thrombosis (DVT) risk screening was done preop- eratively by using a DVT risk screening tool; ${ }^{17)}$ mechanical prophylaxis and aspirin or low-molecular-weight heparin for DVT prophylaxis. Ambulation was started the evening or next morning of surgery, depending on the recovery of quadriceps function. Multimodal pain control was continued with minimal use of narcotics and injectable nonsteroidal anti-inflammatory drugs as rescue analgesia. Usual length of hospital stay was 3 to 4 days.

\section{Follow-up and Outcome Assessment}

All included patients were followed up for 2 years following surgery as per the planned schedule (Fig. 2). A trained physiotherapist (AS) blinded to the type of implant used performed all the measurements as per the standardized guidelines for each outcome. The data were collected on a semi-structured questionnaire after requisite permissions were obtained. New KSS, FJS, and DOPS were assessed and verified by the supervisor (VK) of the study. The patient and assessor were blinded, as the type of prosthesis used was not informed to the patient or the assessor.

\section{Statistical Analysis}

The data were analyzed by using Stata ver. 12 (StataCorp.). The $t$-test was used for evaluation of numerical parametric data. Categorical data were compared by using chi-square test. A $p$-value of $<0.05$ was considered to indicate statistically significant association.

\section{RESULTS}

Demographics, comorbidities, and severity of osteoarthritis were similar between MP and PS groups (Table 1 ). Average age of patients was close to 65 years in both groups with majority being women. In both groups, most patients were American Society of Anesthesiologists grade II. Average deformity (coronal and sagittal together) was $28.25^{\circ} \pm 19.88^{\circ}$ in the PS group and $20.27^{\circ} \pm 10.36^{\circ}$ in the MP group. Although the mean degree of deformity was lower in the MP group, the difference was not statistically significant $(p<0.068)$. On the severity of deformity in the PS group, 13 (32.5\%) had mild, 10 (25\%) had moderate, and $17(42.5 \%)$ had severe deformity, whereas in the MP group, 20 (50\%) had mild, seven (17.5\%) had moderate, and $13(32.5 \%)$ had severe deformity, and difference between two groups was not significant $(p=0.280)$. Baseline mean knee flexion was close to $110^{\circ}$ in both groups (Table 2). DOPS was used to assess performance in each dimension (i.e., timed up and go [TUG] test, stair climb test, selfpaced walk [SPW] test, chair to stand test, and leg holding test) and values were similar between groups. New KSS 
Kulshrestha et al. Medial Pivot vs. Posterior-Stabilized Total Knee Arthroplasty

Clinics in Orthopedic Surgery • Vol. 12, No. 2, $2020 \bullet$ www.ecios.org

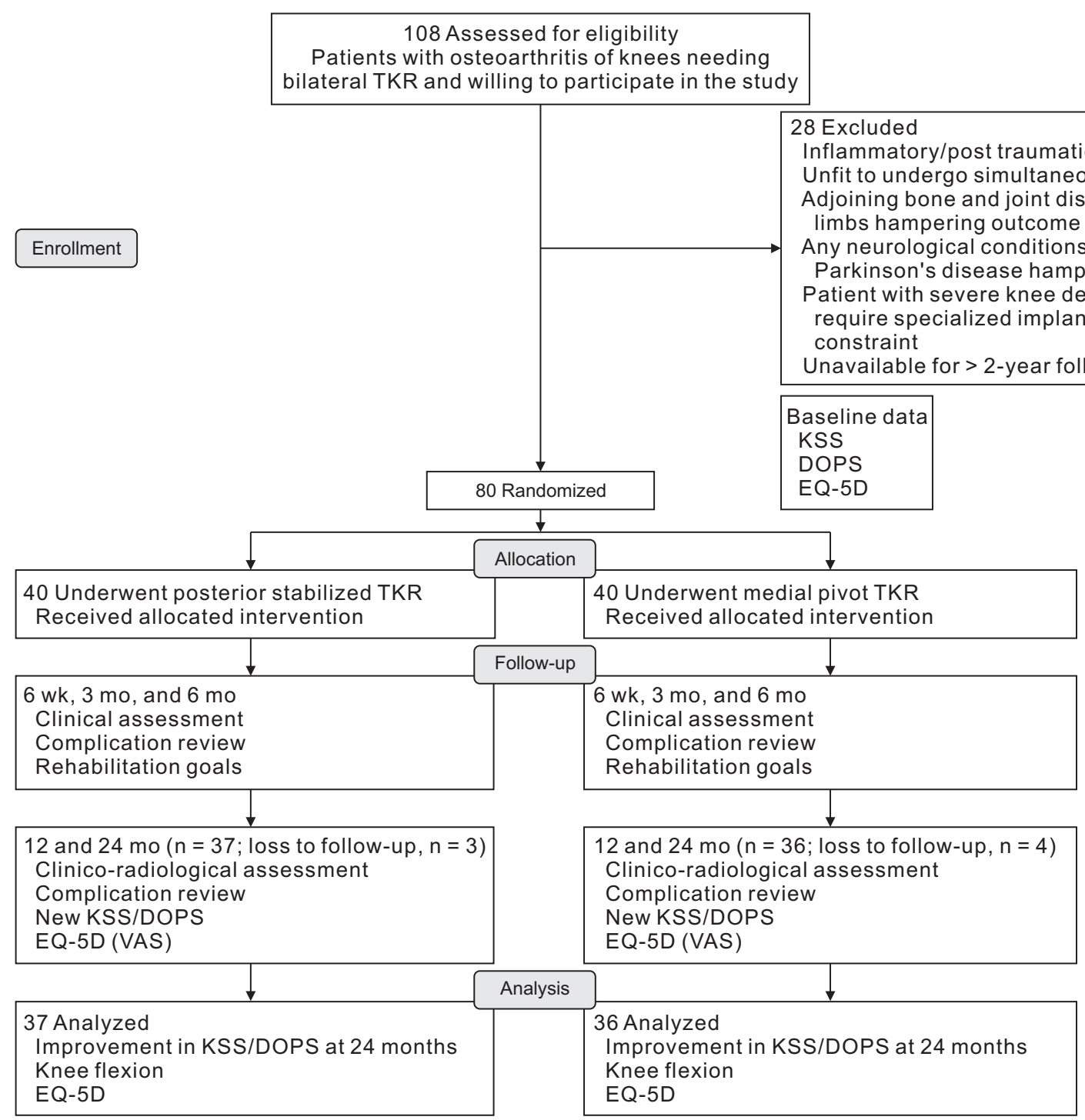

Fig. 2. Consort diagram of patient enrollment, recruitment, and follow-up. TKR: total knee replacement, KSS: Knee Society Score, DOPS: Delaware Osteoarthritis Profile Score, EQ-5D: European Quality of Life Index five dimensional on visual analog scale, VAS:visual analog scale.

(objective, satisfaction, expectation, and activity scores) was not statistically significantly different between groups. FJS-12 was also similar between groups at baseline. General health assessment score (European Quality of Life Index five dimensional on visual analogue scale [EQ-5D]) was also similar between the groups. Thus, preoperatively, patients in two groups were matched statistically for patient-reported activity, function, and performance and were comparable with no potential confounders (Table 2). At 2-year follow-up (Table 2), mean knee flexion was higher in the PS group than in the MP group and the difference was statistically significant $(p=0.013)$. The change in mean knee flexion was $9.25^{\circ} \pm 14.26^{\circ}$ in the PS group as compared to $-6.11^{\circ} \pm 19.74^{\circ}$ in the MP group, thus show- ing worsening of knee flexion in the MP group (Table 2). Statistically, there was a significant difference in the extent of change between two groups $(p=0.004)$. On comparison of the change in different parameters (compared to baseline) of DOPS, significant differences were found in TUG test and SPW only (Table 2). Mean reduction in TUG test was greater in the MP group than in the PS group, whereas the mean increment in SPW test was again greater in the MP group than in the PS group. On the change in different components of new KSS, there was no significant difference between two groups at 24-month follow-up for satisfaction, expectation, and activity scale. However, with respect to the change in scores, the difference between two groups was significant for the objective component only, 
Kulshrestha et al. Medial Pivot vs. Posterior-Stabilized Total Knee Arthroplasty

Clinics in Orthopedic Surgery • Vol. 12, No. 2, $2020 \bullet$ www.ecios.org

Table 1. Patient Demographic Data, Comorbidities, and Disease Severity

\begin{tabular}{|c|c|c|c|c|c|c|}
\hline \multirow{2}{*}{ Variable } & \multicolumn{2}{|c|}{ PS TKR } & \multicolumn{2}{|c|}{ MP TKR } & \multirow{2}{*}{$p$-value } & \multirow{2}{*}{ Effect size } \\
\hline & $\mathrm{n}$ & Mean $\pm S D$ & $\mathrm{n}$ & Mean $\pm S D$ & & \\
\hline Age (yr) & 40 & $65.97 \pm 6.7$ & 40 & $63.8 \pm 6.8$ & 0.155 & 0.43 \\
\hline Sex (male : female) & $17: 23$ & & $11: 29$ & & 0.160 & 0.34 \\
\hline Body mass index $\left(\mathrm{kg} / \mathrm{m}^{2}\right)$ & 40 & $26.64 \pm 4.3$ & 40 & $27.34 \pm 5.1$ & 0.510 & 0.21 \\
\hline Functional comorbidity index & 40 & $0.98 \pm 1.1$ & 40 & $0.58 \pm 0.7$ & 0.154 & 0.41 \\
\hline ASA grade (I : II) & $8: 32$ & & $5: 35$ & & 0.332 & \\
\hline Hemoglobin (\%) & 40 & $11.97 \pm 1.5$ & 40 & $11.92 \pm 0.9$ & 0.858 & 0.04 \\
\hline Average deformity $\left({ }^{\circ}\right)^{*}$ & 40 & $28.25 \pm 19.9$ & 40 & $20.27 \pm 10.4$ & 0.068 & 0.15 \\
\hline Mild & 13 & & 20 & & 0.279 & 0.15 \\
\hline Moderate & 10 & & 7 & & & \\
\hline Severe & 17 & & 13 & & & \\
\hline
\end{tabular}

PS: posterior-stabilized, TKR: total knee replacement, MP: medial pivot, ASA: American Society of Anesthesiologists.

*Total fixed deformity in sagittal and coronal planes.

Table 2. Baseline and Improvement $(\Delta)$ in Patient-Reported Activity, Function, and Performance in the Two Groups

\begin{tabular}{|c|c|c|c|c|c|c|c|}
\hline \multirow{2}{*}{ Variable } & \multicolumn{3}{|c|}{ Baseline } & \multicolumn{3}{|c|}{ Improvement $(\Delta)$} & \multirow{2}{*}{ Effect size } \\
\hline & PS TKR $(n=40)$ & MP TKR $(n=40)$ & $p$-value & PS TKR $(n=37)$ & MP TKR $(n=36)$ & $p$-value & \\
\hline Knee flexion $\left({ }^{\circ}\right)$ & $108.8 \pm 16.4$ & $113.9 \pm 7.7$ & 0.884 & $9.3 \pm 14.3$ & $-6.1 \pm 19.7$ & 0.004 & 0.41 \\
\hline \multicolumn{8}{|l|}{ Delaware index } \\
\hline Timed up and go (sec) & $35.1 \pm 15.0$ & $51.7 \pm 28.5$ & 0.981 & $-21.9 \pm 12.2$ & $-36.2 \pm 7.1$ & 0.026 & 0.58 \\
\hline Stair climb test (sec) & $114.5 \pm 100.1$ & $111.1 \pm 53.0$ & 0.450 & $-89.7 \pm 80.4$ & $-83.0 \pm 59.3$ & 0.613 & \\
\hline Self-paced walk test (m/sec) & $0.4 \pm 0.2$ & $0.8 \pm 0.3$ & 0.764 & $0.5 \pm 0.3$ & $0.9 \pm 0.2$ & 0.002 & 0.61 \\
\hline Chair to stand test & $5.2 \pm 3.1$ & $5.3 \pm 1.8$ & 0.585 & $3.5 \pm 2.4$ & $4.3 \pm 4.8$ & 0.766 & \\
\hline Leg holding test (sec) & $24.0 \pm 14.8$ & $19.1 \pm 9.2$ & 0.115 & $11.7 \pm 27.5$ & $19.7 \pm 16.7$ & 0.855 & \\
\hline \multicolumn{8}{|l|}{ New KSS } \\
\hline Objective (max 100) & $17 \pm 2.8$ & $16.9 \pm 5.9$ & 0.885 & $67.5 \pm 18.8$ & $44.5 \pm 12.3$ & 0.000 & 0.58 \\
\hline Satisfaction (max 40) & $8.4 \pm 4.9$ & $10.2 \pm 3.1$ & 0.052 & $24.2 \pm 6.4$ & $22.6 \pm 6.5$ & 0.220 & \\
\hline Expectation (max 15) & $12.1 \pm 2.6$ & $11.2 \pm 3.5$ & 0.187 & $0.4 \pm 3.0$ & $0.2 \pm 4.0$ & 0.420 & \\
\hline Activity (max 100) & $16.5 \pm 19.3$ & $20.1 \pm 21.2$ & 0.427 & $42.9 \pm 15.6$ & $43.7 \pm 8.4$ & 0.574 & \\
\hline $\mathrm{EQ}-5 \mathrm{D}(\max 100)$ & $31.5 \pm 11.0$ & $36.7 \pm 14.6$ & 0.888 & $53.3 \pm 15.5$ & $47.2 \pm 12.6$ & 0.100 & \\
\hline FJS-12 & $24.2 \pm 4.3$ & $25.2 \pm 8.2$ & 0.685 & $55.6 \pm 15.38$ & $52.7 \pm 14.6$ & 0.276 & \\
\hline
\end{tabular}

Values are presented as mean \pm standard deviation.

PS: posterior-stabilized, TKR: total knee replacement, MP: medial pivot, KSS: Knee Society Score, EQ-5D: European Quality of Life Index five dimensional on visual analog scale, FJS: Forgotten Joint Score.

which was significantly higher in the PS group than in the MP group $(p<0.001)$ because improvement of flexion was significantly greater in the PS group. With respect to the change in EQ-5D and FJS-12 scores, the difference between two groups was not significant statistically at 24 months of follow-up (Table 2). 
In the PS group, one patient had stiff knee (2.5\%) and knee pain and crepitus developed in two patients (5\%). In the MP group, wound complication was noted as the complication in one patient (2.5\%). Statistically, there was no significant difference between two groups with respect to complications $(p>0.05)$. None of the patients in either group had prosthetic joint infection, instability, cardiopulmonary complications, or periprosthetic fracture.

\section{DISCUSSION}

The search for exclusive knee design, which mimics native mechanics, restores optimum function, ensures longevity, and meets all expectations of our increasingly younger clientele, continues. Despite improvement in all aspects of TKA, there remains a significant subset of patients who are dissatisfied. Patient-reported satisfaction is multidimensional; knee implant design is one domain that has seen recent advances to optimize patient outcomes. Our study attempted to compare outcomes of one such design, MP knee, with the conventional PS knee. This design was developed to enhance stability and reduce wear by creating a near constant femoral component radius to mimic normal knee kinematics. As far as MP total knee arthroplasty is concerned, there are limited studies comparing its outcomes with those of other implant designs (Table $3)^{5,13,18-25)}$ To date, there is only one randomized study by Hossain et al., which has compared outcomes of an MP knee to a fixed-bearing multi-radius PS implant. There are three other randomized controlled trials (RCTs), which have compared an MP design to a mobile PS knee: an ultra-congruent rotating platform knee in studies by Kim et al. ${ }^{18)}$ and Kim et al. ${ }^{19)}$ and a single radius knee in a study by Benjamin et al. ${ }^{20)}$ There are a few more matched pair studies and case series, which have looked at outcomes of the MP design.

The present randomized study was an attempt (1) to analyze the outcomes of an MP knee compared to a widely

Table 3. Review of Available Literature Comparing Outcomes of Medial Pivot vs. Other Designs

\begin{tabular}{|c|c|c|c|c|c|c|c|}
\hline \multirow{2}{*}{ Study type } & \multirow{2}{*}{ Study } & \multirow{2}{*}{$\begin{array}{l}\text { Follow-up } \\
\text { (yr) }\end{array}$} & \multirow{2}{*}{$\begin{array}{c}\text { MP TKA/ } \\
\text { other design }\end{array}$} & \multicolumn{4}{|c|}{ Outcome assessed (MP vs. other design) } \\
\hline & & & & ROM & PROM & Performance & General health \\
\hline \multicolumn{8}{|l|}{ Retrospective } \\
\hline Matched pair & Bae et al. $(2016)^{13)}$ & 5 & 150/150 (PS) & Better in PS & $\begin{array}{l}\text { No difference } \\
\text { (KSS \& WOMAC) }\end{array}$ & $\begin{array}{l}\text { Better in MP } \\
\text { (Kujala \& Feller) }\end{array}$ & - \\
\hline Matched pair & Nakamura $(2018)^{25)}$ & 2 & 45/45 (CR) & Better in MP & Better in MP (KSS) & - & - \\
\hline Case series & Samy $(2018)^{22)}$ & 1 & 76/88 (PS) & ND & Better in MP (FJS) & - & - \\
\hline Case series & Shakespeare $(2006)^{24)}$ & 1 & 261/288 (PS) & ND & - & - & - \\
\hline Case series & Choi et al. $(2017)^{23)}$ & 5 & 49/52 (UC/RP) & ND & $\begin{array}{l}\text { No difference } \\
\text { (KSS \& WOMAC) }\end{array}$ & - & - \\
\hline \multicolumn{8}{|l|}{ Prospective } \\
\hline $\mathrm{RCT}$ & Hossain et al. $(2011)^{5)}$ & 2 & 40/40 (PS) & Better in MP & $\begin{array}{l}\text { Better in MP } \\
\text { (WOMAC/Pain) }\end{array}$ & $\begin{array}{l}\text { Better in MP } \\
\text { (TKFO) }\end{array}$ & $\begin{array}{l}\text { Better physical } \\
\text { in MP (SF-36) }\end{array}$ \\
\hline $\mathrm{RCT}$ & Kim et al. (2009) & 2 & 92/92 (PS mobile) & Better in PS & Better in PS (KSS) & Better in PS (HSS) & - \\
\hline RCT & Kim et al. $(2017)^{19)}$ & 11 & 182/182 (UC/RP) & Better in UC & $\begin{array}{l}\text { Better in PS } \\
\text { (KSS \& WOMAC) }\end{array}$ & - & - \\
\hline $\mathrm{RCT}$ & Benjamin $(2018)^{20)}$ & 2 & $\begin{array}{l}\text { 45/45 (PS-single } \\
\text { radius) }\end{array}$ & - & ND (KSS) & - & - \\
\hline Cohort study & Papagiannis $(2016)^{21)}$ & & 24/22 (UC/RP) & ND & ND (KSCS) & $\mathrm{ND}(\mathrm{KFS})$ & - \\
\hline RCT & This study (2018) & 2 & $37 / 36$ (PS) & Better in PS & $\begin{array}{l}\text { ND FJS/better } \\
\text { objective KSS in PS }\end{array}$ & $\begin{array}{l}\text { Better in MP } \\
\text { (SPW \& TUG) }\end{array}$ & ND (E0-5D) \\
\hline
\end{tabular}

MP: medial pivot, TKA: total knee arthroplasty, ROM: range of motion, PROM: patient-reported outcome measures, PS: posterior-stabilized, KSS: Knee Society Score, WOMAC: Western Ontario and McMaster Universities osteoarthritis index, CR: cruciate-retaining, ND: no difference, FJS: Forgotten Joint Score, UC/RP: ultracongruent/rotating platform insert, RCT: randomized controlled trial, TKFO: total knee function questionnaire, SF-36: 36-item short form health survey, HSS: Hospital for Special Surgery Score, KSCS: Knee Society Clinical Score, KFS: Knee Function Score, SPW \& TUG: self-paced walk and timed up and go, EQ-5D: European Quality of Life Index five dimensional on visual analog scale. 
Kulshrestha et al. Medial Pivot vs. Posterior-Stabilized Total Knee Arthroplasty

Clinics in Orthopedic Surgery • Vol. 12, No. 2, $2020 \bullet$ www.ecios.org

used contemporary knee design, a fixed bearing PS knee, (2) and to see if changes in design can affect outcomes. Our study used a comprehensive set of outcome assessment tools, which included objective assessment of knee flexion, patient-reported outcomes using new KSS, high functionality index using FJS, and objective measure of patient performance using DOPS.

In our study, MP group patients, at 24 months of follow-up, as judged by DOPS, had significantly better improvement in the TUG test (reduction of 36 seconds as compared to 22 seconds in PS group; $p<0.026$ ) and SPW test (improvement by $0.9 \mathrm{~m} / \mathrm{sec}$ in the MP group as compared to $0.5 \mathrm{~m} / \mathrm{sec}$ in the PS group; $p<0.002$ ). This could be attributed to the single radius curvature and high conformity in the medial compartment. Bae et al., ${ }^{13)}$ also reported significantly better Kujala and Feller scores, which assess quadriceps strength and activity such as getting up from chair, stair climbing, walking, running, and jumping. Similar to our and Bae et al.s studies, ${ }^{13)}$ the RCT done by Hossain et al. ${ }^{5)}$ found improvement in patient performance in terms of activities of daily living and high-performance activities like sports and exercises were significantly better in the MP group than in the PS knee. According to Papagiannis et al.s kinematic and kinetic analysis, ${ }^{21)}$ early outcomes could not demonstrate any notable functional and wear advantages between the two groups. Likewise, Benjamin et al.s study ${ }^{20)}$ revealed comparable functional grades for both PS and MP groups. However, a level I RCT by Kim et al. ${ }^{18)}$ showed strong preferences for the non-MP implants as they obtained smaller ranges of knee motion, less patient satisfaction (Hospital for Special Surgery Score, $p=0.023$ ), and a higher complication rate in fixed bearing MP prostheses in their early outcome, despite similar long-term fixation and survival rates of both MP and PFC Sigma prostheses in their subsequent report. ${ }^{19)}$

In our study, when we looked at knee flexion, the maximum flexion achieved was significantly better in patients who had PS knees than in MP knees $\left(118^{\circ}\right.$ vs. $108^{\circ}$, $p<0.011)$. When we looked at the gain in flexion, the PS group patients gained an average of $9^{\circ}$ as compared to the MP group who lost an average of $6^{\circ}$ of motion compared to baseline $(p<0.004)$. Our results compare well with those of Kim et al. ${ }^{18,19)}$ who studied the MP knee to compare it with the mobile bearing PS device. They found final flexion achieved was better in PS knees than in MP knees. Similarly, Bae et al., ${ }^{13)}$ in their matched pair study of $150 \mathrm{MP}$ vs. PS knees, found flexion to be better in the PS design. However, Hossain et al., ${ }^{5}$ in their RCT, found final flexion was better in the MP design. We already know that the flexion achieved by the patients undergoing TKA is multifactorial and prosthetic design may not be the only determining factor. However, it appears that the PS design with its predictive rollback may facilitate better flexion in most patients, thus providing better objective scores in patient-reported outcomes.

Patient-reported outcome scores we used (KSS and FJS) have objective and subjective components. In our study, PS knee patients had a better KSS, which was due to better objective scores recorded as a result of improved flexion. The FJS was similar in both groups as in the study by Kim et al. ${ }^{18)}$ Contrary to our findings, Hossain et al. ${ }^{5)}$ in their RCT and Samy et al. ${ }^{22)}$ in their matched pair study found better WOMAC score and FJS score in MP knees as compared to PS knees. Various studies ${ }^{13,20,21,23)}$ found no difference in patient-reported outcome measures (PROM) when they compared an MP knee to other designs. It seems that knee flexion was the prime determinant in deciding final PROM scores, and hence the PROM scores favored devices that facilitated better flexion in a patient.

Contrary to results published by Kim et al., ${ }^{18,19)}$ our study was devoid of complications of deep infections and recurrent effusions in either group. Samy et al. ${ }^{22)}$ described marginally higher incidence of instability rates in the MP prosthesis than in PS knees $(2: 1)$. As acknowledged by Shakespeare et al., ${ }^{24)}$ we also found most MP knees to be inherently stable, which was an objective assessment of stability by conventional methods of evaluation of achieving equal medial and lateral gaps through $0^{\circ}$ to $90^{\circ}$ flexion intraoperatively. In our series, among the PS knee, one patient received blood transfusion for postoperative dip in hematocrit, another one was manipulated for stiff knee, whereas two were managed conservatively for anterior knee pain and crepitus. Parallel to findings by Minoda et $\mathrm{al}^{26)}$ of production of fewer wear particles in MP prosthesis leading to reduced incidence of osteolysis and aseptic loosening, none of our study group patients developed implant loosening at the end of study.

Our study has a few limitations. First, we did not analyze postoperative radiographs for implant fixation and alignment. However, the combined subjective and objective components of KSS apparently took limb alignment into consideration, and quality of cementing as assessed by the radiographs is unlikely to affect functional outcomes. Second, the 24-month follow-up was relatively short; however, patients with uneventful recovery are likely to have achieved optimum performance by this duration of follow-up. Third, we studied the difference in outcomes of two implant designs with different insert geometry and commensurate difference in femoral component anatomy, hence it is not possible to determine whether the differ- 
Kulshrestha et al. Medial Pivot vs. Posterior-Stabilized Total Knee Arthroplasty

Clinics in Orthopedic Surgery • Vol. 12, No. 2, $2020 \bullet$ www.ecios.org

ence in outcome was due to the single radius femur or MP insert geometry. However, we compared outcomes of two different successful implant designs. The strengths of our study include its method of randomization and concealment of treatment allocation; the outcome assessment was well blinded by using an independent assessor who was unaware of the type of implant used. A priori calculation of sample size and low attrition (10\%) rate at followup gave adequate strength to conclusions drawn. We used a comprehensive set of validated outcome assessment tools, which allowed us to compare the two groups in a meticulous manner. Using patients with bilateral disease undergoing bilateral surgery facilitated easy comparison between the groups without the need for controlling the disease severity of the unoperated side. Finally, as the senior operating surgeon was well trained in use of both the implant designs and its instrumentation, it ensured surgical equipoise.

The MP knee offers advantages to the patients who wish to perform certain activities of daily living requiring good quadriceps strength and balance. Getting up from chair and walking speed in MP knee patients were significantly better than those in PS knee patients. At the same time, due to predictable rollback ensured by cam and post, the PS knee facilitated better average knee flexion. However, the patients did not perceive the relative superiority; they were equally satisfied with both designs.

\section{CONFLICT OF INTEREST}

No potential conflict of interest relevant to this article was reported.

\section{ACKNOWLEDGEMENTS}

Mrs. Pramila Sharma (BSc in Nursing, Department of Orthopaedics, Air Force Hospital, Kanpur) was the study nurse coordinator. Mr. A Shinoj (Diploma in Physiotherapy, Department of Orthopaedics, Air Force Hospital, Kanpur) carried out enrollment counseling and blinded outcome assessment for the study.

\section{REFERENCES}

1. Hooper G, Lee AJ, Rothwell A, Frampton C. Current trends and projections in the utilisation rates of hip and knee replacement in New Zealand from 2001 to 2026. N Z Med J. 2014;127(1401):82-93.

2. Dunbar MJ, Richardson G, Robertsson O. I can't get no satisfaction after my total knee replacement: rhymes and reasons. Bone Joint J. 2013;95(11 Suppl A):148-52.

3. Choi YJ, Ra HJ. Patient satisfaction after total knee arthroplasty. Knee Surg Relat Res. 2016;28(1):1-15.

4. Atzori F, Salama W, Sabatini L, Mousa S, Khalefa A. Medial pivot knee in primary total knee arthroplasty. Ann Transl Med. 2016;4(1):6.

5. Hossain F, Patel S, Rhee SJ, Haddad FS. Knee arthroplasty with a medially conforming ball-and-socket tibiofemoral articulation provides better function. Clin Orthop Relat Res. 2011;469(1):55-63.

6. Bordini B, Ancarani C, Fitch DA. Long-term survivorship of a medial-pivot total knee system compared with other cemented designs in an arthroplasty registry. J Orthop Surg Res. 2016;11:44.

7. Brinkman JM, Bubra PS, Walker P, Walsh WR, Bruce WJ. Midterm results using a medial pivot total knee replacement compared with the Australian National Joint Replacement Registry data. ANZ J Surg. 2014;84(3):172-6.
8. Fan CY, Hsieh JT, Hsieh MS, Shih YC, Lee CH. Primitive results after medial-pivot knee arthroplasties: a minimum 5-year follow-up study. J Arthroplasty. 2010;25(3):492-6.

9. Chinzei N, Ishida K, Tsumura N, et al. Satisfactory results at 8 years mean follow-up after ADVANCE ${ }^{\circledR}$ medial-pivot total knee arthroplasty. Knee. 2014;21(2):387-90.

10. Fitch DA, Sedacki K, Yang Y. Mid- to long-term outcomes of a medial-pivot system for primary total knee replacement: a systematic review and meta-analysis. Bone Joint Res. 2014;3(10):297-304.

11. Karachalios T, Roidis N, Giotikas D, Bargiotas K, Varitimidis S, Malizos KN. A mid-term clinical outcome study of the Advance Medial Pivot knee arthroplasty. Knee. 2009;16(6):484-8.

12. Macheras GA, Galanakos SP, Lepetsos P, Anastasopoulos PP, Papadakis SA. A long term clinical outcome of the Medial Pivot Knee Arthroplasty System. Knee. 2017;24(2):44753.

13. Bae DK, Cho SD, Im SK, Song SJ. Comparison of midterm clinical and radiographic results between total knee arthroplasties using medial pivot and posterior-stabilized prosthesis: a matched pair analysis. J Arthroplasty. 2016;31(2):41924.

14. Mizner RL, Petterson SC, Clements KE, Zeni JA Jr, Irrgang 
Kulshrestha et al. Medial Pivot vs. Posterior-Stabilized Total Knee Arthroplasty

Clinics in Orthopedic Surgery • Vol. 12, No. 2, $2020 \bullet$ www.ecios.org

JJ, Snyder-Mackler L. Measuring functional improvement after total knee arthroplasty requires both performancebased and patient-report assessments: a longitudinal analysis of outcomes. J Arthroplasty. 2011;26(5):728-37.

15. Lizaur-Utrilla A, Gonzalez-Parreno S, Martinez-Mendez D, Miralles-Munoz FA, Lopez-Prats FA. Minimal clinically important differences and substantial clinical benefits for Knee Society Scores. Knee Surg Sports Traumatol Arthrosc. 2019 May 20 [Epub]. https://doi.org/10.1007/s00167-01905543-x.

16. Kulshrestha V, Kumar S, Datta B, Sinha VK, Mittal G. Ninety-day morbidity and mortality in risk-screened and optimized patients undergoing two-team fast-track simultaneous bilateral TKA compared with unilateral TKA: a prospective study. J Arthroplasty. 2018;33(3):752-60.

17. Kulshrestha V, Kumar S. DVT prophylaxis after TKA: routine anticoagulation vs risk screening approach: a randomized study. J Arthroplasty. 2013;28(10):1868-73.

18. Kim YH, Yoon SH, Kim JS. Early outcome of TKA with a medial pivot fixed-bearing prosthesis is worse than with a PFC mobile-bearing prosthesis. Clin Orthop Relat Res. 2009;467(2):493-503.

19. Kim YH, Park JW, Kim JS. Clinical outcome of medial pivot compared with press-fit condylar sigma cruciate-retaining mobile-bearing total knee arthroplasty. J Arthroplasty. 2017;32(10):3016-23.

20. Benjamin B, Pietrzak JR, Tahmassebi J, Haddad FS. A functional comparison of medial pivot and condylar knee designs based on patient outcomes and parameters of gait. Bone Joint J. 2018;100(1 Supple A):76-82.

21. Papagiannis GI, Roumpelakis IM, Triantafyllou AI, Makris IN, Babis GC. No differences identified in transverse plane biomechanics between medial pivot and rotating platform total knee implant designs. J Arthroplasty. 2016;31(8):181420.

22. Samy DA, Wolfstadt JI, Vaidee I, Backstein DJ. A retrospective comparison of a medial pivot and posterior-stabilized total knee arthroplasty with respect to patient-reported and radiographic outcomes. J Arthroplasty. 2018;33(5):1379-83.

23. Choi NY, In Y, Bae JH, Do JH, Chung SJ, Koh IJ. Are midterm patient-reported outcome measures between rotatingplatform mobile-bearing prosthesis and medial-pivot prosthesis different? A minimum of 5-year follow-up study. J Arthroplasty. 2017;32(3):824-9.

24. Shakespeare D, Ledger M, Kinzel V. Flexion after total knee replacement: a comparison between the Medial Pivot knee and a posterior stabilised implant. Knee. 2006;13(5):371-3.

25. Nakamura J, Inoue T, Suguro T, et al. A comparative study of flat surface design and medial pivot design in posterior cruciate-retaining total knee arthroplasty: a matched pair cohort study of two years. BMC Musculoskelet Disord. 2018;19(1):234.

26. Minoda Y, Kobayashi A, Iwaki H, et al. Polyethylene wear particles in synovial fluid after total knee arthroplasty. Clin Orthop Relat Res. 2003;(410):165-72. 